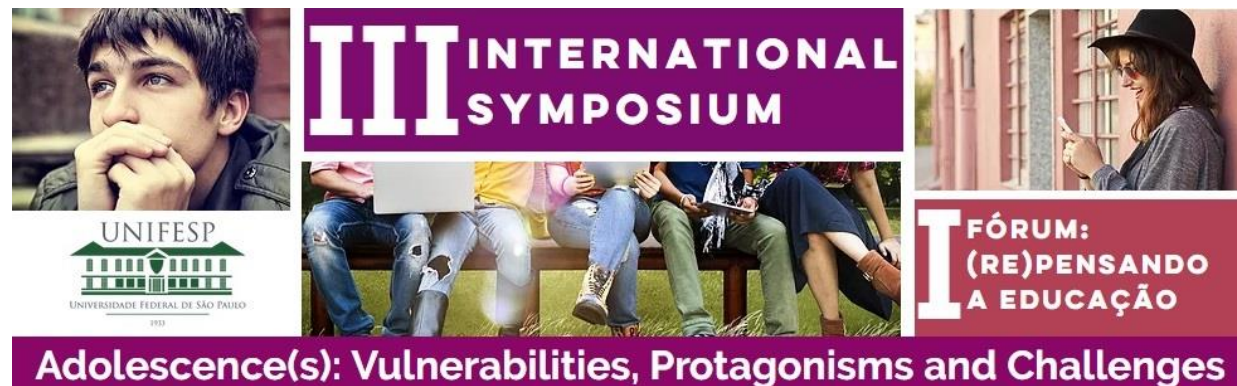

THE ACCURACY AND THE UTILIZATION OF THE PUBERTAL DEVELOPMENT SCALE, A SELF-EVALUATION SCALE PUBERTAL STAGING

\title{
ADAPTAÇÂO PARA USO NO BRASIL E ACURÁCIA DA PUBERTAL DEVELOPMENT $S C A L E$, UMA ESCALA DE AUTOAVALIAÇÃO DE ESTADIAMENTO PUBERAL
}

Rafaella Sales de Freitas ${ }^{1}$; Luanna Inacio ${ }^{2}$; Gislaine Valverde Zanini ${ }^{3}$; Giovana Ribeiro de Souza $^{4}$; Flávia Calanca da Silva ${ }^{5}$; Maria Sylvia de Souza Vitalle ${ }^{6}$; Sheila Rejane Niskier ${ }^{7}$; Sabine Pompéia ${ }^{8}$

1. Graduanda de Ciências Biomédicas na Universidade Federal de São Paulo

E-mail: rafs2freitas@gmail.com

2. Graduanda de Ciências Biomédicas na Universidade Federal de São Paulo E-mail: luannamaristella@gmail.com

3. Doutoranda do Departamento de Psicobiologia, Universidade Federal de São Paulo

E-mail: giavz@yahoo.com.br

4. Médica residente em Medicina do Adolescente, Universidade Federal de São Paulo

E-mail: giovanards@gmail.com

5. Médica Técnica Administrativa Universidade Federal de São Paulo

E-mail: calanca.flavia@gmail.com

6. Professora adjunta do Departamento de Pediatria, Universidade Federal de São Paulo

E-mail: sylviavitalle@gmail.com

7. Médica Técnica Administrativa Universidade Federal de São Paulo

E-mail: sheilaniskier@uol.com.br

8. Professora adjunta do Departamento de Psicobiologia Universidade Federal de São Paulo E-mail: spompeia@gmail.com

* Iniciação científica desenvolvida no Departamento de Psicobiologia, Universidade Federal de São Paulo

\section{Como citar:}

FREITAS, Rafaella Sales de et al. The accuracy and the utilization of the pubertal development scale, a selfevaluation scale pubertal staging. In: INTERNATIONAL SYMPOSIUM ADOLESCENCE(S) \& FÓRUM (RE)PENSANDO A EDUCAÇÃO, 3, 2017, São Paulo. Anais... São Paulo: Unifesp, 2017. p. 94-95. DOI: http://dx.doi.org/10.22388/2525-5894.2017.045

Introdução: A adolescência se inicia com a puberdade, associada a alterações corporais e comportamentais. O padrão ouro para avaliar o estadiamento puberal é o método de Tanner, 
que classifica jovens em um de 5 estágios de desenvolvimento de pelos pubianos (ambos os sexos) e genitália masculina ou mamas. Examinar genitais e mamas, embora de extrema importância para avaliar possíveis afecções e grau de maturação sexual, muitas vezes constrange os jovens. A avaliação do estadiamento puberal tem ainda, como desvantagem, a necessidade de envolver profissional de saúde treinado e local apropriado para examinar os adolescentes. Uma alternativa empregada internacionalmente é o uso de instrumentos de autoavaliação, como a Escala de Desenvolvimento Puberal (Pubertal Development Scale; PDS). A PDS inquere sobre crescimento de pelos pubianos/corporais, mudanças na pele e estirão de crescimento (ambos os sexos), engrossamento da voz e pelos no rosto (garotos) e mamas e menarca (garotas). Não há versões dessa escala para uso no Brasil.

Objetivo: Neste trabalho foi estudada a correspondência entre o estagiamento pela PDS (adaptada para uso local) com o obtido na avaliação clínica pelo método de Tanner.

Métodos: Participaram do estudo 111 jovens (61 do sexo masculino) com idades 9 e 17 anos atendidos no Centro de Atendimento e Apoio ao Adolescente da Unifesp/HSP. Os escores obtidos pela PDS (variável contínua) e a classificação dos estágios de Tanner (variável ordinal) foram comparados com correlações lineares de Spearman.

Resultados e Discussão: As correlações entre os escores na escala PDS com mamas/genitália e pelos pubianos na escala de Tanner foram, respectivamente: $r=0,75$ e $r=0,70$ (toda a amostra), $\mathrm{r}=0.73$ e $\mathrm{r}=0,75$ (garotos) e $\mathrm{r}=0,79$ e $\mathrm{r}=0,64$ (garotas).

Conclusões: A versão Brasileira da PDS pode ser uma alternativa para realizar o estadiamento puberal quando a avaliação clínica não é viável, sendo útil em diversos contextos que envolvam jovens em desenvolvimento.

Palavras-Chave: Puberdade. Estadiamento puberal. Autoavaliação. 